\title{
Update on the role of eslicarbazepine acetate in the treatment of partial-onset epilepsy
}

This article was published in the following Dove Press journal:

Neuropsychiatric Disease and Treatment

23 May 2016

Number of times this article has been viewed

\section{Renato Tambucci' \\ Claudia Basti' \\ Maria Maresca' \\ Giangennaro Coppola ${ }^{2}$ \\ Alberto Verrotti ${ }^{1}$ \\ 'Department of Pediatrics, University of L'Aquila, L'Aquila, Italy; ${ }^{2}$ Child and \\ Adolescent Neuropsychiatry Unit, \\ University of Salerno, Salerno, Italy}

Correspondence: Alberto Verrotti Department of Pediatrics, University of L'Aquila, via Lorenzo Natali I,

67100, L'Aquila, Italy

Tel +39862 368607

Fax +39862 368726

Email averrott@unich.it
Abstract: Eslicarbazepine acetate (ESL) is a once daily new third generation antiepileptic drug that shares the basic chemical structure of carbamazepine and oxcarbazepine - a dibenzazepine nucleus with the 5-carboxamide substituent, but is structurally different at the 10,11-position. ESL is a pro-drug metabolized to its major active metabolite eslicarbazepine. Despite the fact that the exact mechanism of action has not been fully elucidated, it is thought to involve inhibition of voltage-gated sodium channels (VGSC). ESL inhibits sodium currents in a voltage-dependent way by an interaction predominantly with the inactivated state of the VGSC, thus selectively reducing the activity of rapidly firing (epileptic) neurons. ESL reduces VGSC availability through enhancement of slow inactivation. In Phase III studies, adjunctive therapy with ESL 800 or $1,200 \mathrm{mg} /$ day leads to a significant decrease in the seizure frequency in adults with refractory partial onset epilepsy. Based on these results, ESL has been approved in Europe (by the European Medicines Agency) and in the United States (by the US Food and Drug Administration) as add-on therapy. Data on efficacy and safety have been confirmed by 1 -year extension and real life observational studies. Recently, based on results from two randomized, double-blind, historical control Phase III trials, ESL received US Food and Drug Administration approval also as a monotherapy for patients with partial onset epilepsy. In the pediatric setting, encouraging results have been obtained suggesting its potential role in the management of epileptic children. Overall ESL was generally well tolerated. The most common adverse events were dizziness, somnolence, headache, nausea, diplopia, and vomiting. Adverse events can be minimized by appropriate titration. In conclusion, ESL seems to overcome some drawbacks of the previous antiepileptic drugs, suggesting a major role of ESL in the management of focal onset epilepsy for both new onset and refractory cases, either as monotherapy or as adjunctive treatment.

Keywords: eslicarbazepine, partial-onset epilepsy, antiepileptic drugs, refractory epilepsy

\section{Introduction}

Epilepsy is a chronic disorder of the brain that affects people worldwide. Approximately 50 million people currently suffer from epilepsy, and there are between 30 and 50 cases of new-onset epilepsy per 100,000 people every year. The overall estimated prevalence ranges between four and ten per 1,000 people with a much higher proportion in low and middle income countries (between seven and 14 per 1,000 people). ${ }^{1}$ Despite the fact that most children and adults with epilepsy can be successfully treated with antiepileptic drugs (AEDs), it has been estimated that $22.5 \%$ to $30 \%$ of patients have drug-resistant epilepsy. ${ }^{2}$ Several mechanisms of drug resistance have been suggested, but overall they are likely to be multifactorial according to the underlying cause and to the drug's site of action. ${ }^{3}$ The newer drugs have improved outcomes for people with epilepsy, but this improvement relates mostly to a reduced toxicity burden and fewer adverse drug interactions, with overall no more than $10 \%-15 \%$ of patients refractory 
to older drugs achieving sustained seizure freedom with the newer agents. ${ }^{4,5}$ Overcoming AEDs' resistance represents a challenge and justifies the need to channel the efforts of the scientific community towards the development of novel antiepileptic treatments. New AEDs should be both effective and well tolerated as the adverse effects of AEDs may have a negative impact on quality of life, and can be a significant cause of morbidity ${ }^{6}$ that could lead to discontinuation or reduced adherence to the therapy. ${ }^{7,8}$

Moreover, simplification of drug regimens with once daily therapies, leading to an increased adherence, could result in an improvement in patient outcomes. ${ }^{9}$

Eslicarbazepine acetate (ESL) is a once daily new third generation AED belonging to the dibenzazepine carboxamide chemical class of AEDs. As for carbamazepine (CBZ), and oxcarbazepine (OXC), ESL contains a dibenzazepine nucleus bearing a 5-carboxamide substitute, but it is structurally different at the 10,11 position. ${ }^{10,11}$

ESL was approved in 2009 by the European Medicines Agency (EMA) (Zebinix $\left.{ }^{\mathrm{TM}}\right)^{12}$ and in 2013 by the US Food and Drug Administration (FDA) (Aptiom $\left.{ }^{\mathrm{TM}}\right)^{13}$ as an adjunctive therapy in adults with refractory partial-onset seizures (POS), with or without secondary generalization. In August 2015, the FDA approved the new indication that allows ESL to be used as monotherapy in people who initiate treatment for the first time or convert from other AEDs to ESL. ${ }^{14}$

\section{Methods}

Electronic databases MEDLINE and EMBASE were systematically searched to identify relevant studies published up to February 2016. The search was performed with the use of the following terms: "Eslicarbazepine", "Eslicarbazepine acetate", "Eslicarbazepine AND partial-onset epilepsy", "partial-onset epilepsy". Only full-length publications in English language were included. Reference lists of the selected articles were used to identify further relevant papers. Additional information was also obtained from the websites of US and EU agencies (FDA and EMA).

This review study aimed to provide critical findings of the available literature about the role of ESL in the treatment of partial-onset epilepsy.

\section{Mechanisms of action and pharmacokinetics Mechanisms of action}

ESL ((S)-10-acetoxy-10,11-dihydro-5H-dibenz/b,f/azepine5-carboxamide, with development code BIA 2-093) is a once daily AED. Like CBZ and OXC, ESL has the basic chemical structure of a dibenzazepine nucleus with the 5-carboxamide substituent, but is structurally different at the 10,11-position. ${ }^{10,11}$

The exact mechanism of anticonvulsant action has not been fully elucidated; like CBZ, lamotrigine, OXC, and phenytoin, it is thought that ESL might act by the inhibition of voltage-gated sodium channels (VGSC). ${ }^{15,16}$ The affinity of VGSC blocker drugs for the ion channels differs according to the action on the three distinct functional states of the channel: resting state (the channel is closed but responsive to voltage changes), open state (activated), and inactivated state (closed and not responsive to voltage changes). ESL inhibits sodium currents in a voltage-dependent way by an interaction predominantly with the inactivated state and competitively interacts with neurotoxin receptor site 2 of the VGSC, but not with receptor site 1 , stabilizing the inactive form of the sodium channel. ${ }^{17}$ An important difference between AEDs acting on VGSV is that ESL, as well as lacosamide (LCM), potentially reduce VGSC availability through enhancement of slow inactivation, instead of altering fast inactivation of VGSC as promoted by CBZ and OXC. Compared to ESL, LCM showed higher interaction with VGSC in the resting state, with fast inactivation gating and shorter time to enter into the slow inactivated state. ${ }^{18}$

This suggests that ESL is less likely to bind to normally active neurons, suggesting a similar effect on sustained repetitive neural firing with a lower neurological toxicity. ${ }^{10,19}$

ESL also blocks high- and low-affinity Cav3.2 inward currents with greater affinity than CBZ, but without effect on voltage-gated Kv7.2 potassium channels, in contrast with CBZ that reduces the outward Kv7.2 potassium current; another new generation AED, retigabine, a Kv7.2/Kv7.3 opener, acts by increasing open channels, leading to hyperpolarization of the membrane potential. ${ }^{18,20,21}$

\section{Pharmacokinetics}

ESL is a pro-drug that is metabolized to its major active metabolite eslicarbazepine (S-licarbazepine that represents $95 \%$ of circulating active moieties) and to the minor active metabolites R-licarbazepine and oxcarbazepine. Eslicarbazepine is responsible for pharmacological effects. ${ }^{15,16}$

After oral administration, absorption of ESL from the gastrointestinal tract is high. In fact, the amount of metabolites recovered in urine corresponds to more than $90 \%$ of the administered ESL dose. ${ }^{16}$ Consequently, plasma eslicarbazepine concentration always remains below the limit of detection. ${ }^{15}$ Food does not significantly affect the pharmacokinetics of ESL. ${ }^{22}$ Steady state is reached within 4-5 days with once daily administration of ESL. ${ }^{23}$ Pharmacokinetic/pharmacodynamic analysis demonstrates that as the ESL dosage and eslicarbazepine concentrations increase, the seizure frequency decreases. ${ }^{24}$ 
Plasma protein binding of eslicarbazepine is relatively low $(40 \%)$ and is not affected by other coadministered drugs. ${ }^{15}$ Plasma protein binding of eslicarbazepine is not affected to a clinically relevant extent by warfarin, diazepam, digoxin, phenytoin or tolbutamide, and the plasma protein binding of these agents is not affected by ESL. Metabolites of ESL are mainly eliminated from the systemic circulation by renal excretion: approximately two thirds of them are in the unchanged form and one third as glucuronide conjugates. ${ }^{12-14}$

Plasma concentrations of eslicarbazepine decline in a multiphasic manner, with a terminal elimination half-life of 13-20 hours in patients with POS who received ESL 400, 800 or $1,200 \mathrm{mg}$ once daily. ${ }^{25}$

Sex does not influence the pharmacokinetics of ELS, and no differences have been observed in the ESL pharmacokinetics profile between young healthy subjects (aged $18-40$ years) and elderly healthy subjects (aged $>65$ years) after both single and repeated administration of ESL. ${ }^{22,26}$

\section{Drug interactions}

Drug interactions can be significant: in fact, ESL can have an inducing effect on the metabolism of drugs that are primarily eliminated by metabolism through CYP3A4 (CBZ, phenobarbital, topiramate) or conjugation through the UDP glucuronosyl (lamotrigine). ${ }^{16}$

Thus, an increase in the dose of these drugs that are mainly metabolized through CYP3A4 or through the UDPglucuronosyl transferases may be required when coadministered with ESL. ${ }^{16}$

Moreover, eslicarbazepine has inhibiting properties against CYP2C9 and CYP2C19 and, then, can interact in cotreatment with drugs metabolized primarily by CYP2C9 and CYP2C19 (phenytoin). ${ }^{16}$

\section{Interactions with other AEDs}

Data from literature demonstrate an interaction of ESL with other AEDs, such as CBZ, lamotrigine, topiramate, and phenytoin. ${ }^{12-14,27-29}$ The main interactions are reported in the next paragraphs.

\section{CBZ}

Eslicarbazepine exposure decreases when CBZ is coadministered with $\mathrm{ESL}^{30}$ and, based on the individual response, the ESL dosage may need to be increased when coadministered with CBZ. ${ }^{12-14,27-29}$

\section{Lamotrigine}

Coadministration of ESL 1,200 mg once daily with lamotrigine $150 \mathrm{mg}$ once daily decreased lamotrigine exposure (which is also mainly metabolized by glucuronidation) by $14 \%, 27$ therefore, no dosage adjustment is required in patients receiving concomitant ESL and lamotrigine. ${ }^{12-14}$

\section{Topiramate}

An $18 \%$ reduction in topiramate exposure occurred when administered in combination with ESL 1,200 mg once daily. Therefore, no dosage adjustment is required..$^{28}$

\section{Phenytoin}

A concomitant administration of ESL with phenytoin produces an average increase of $31 \%-35 \%$ in exposure to phenytoin, most likely caused by an inhibition of CYP2C9 and CYP2C19; consequently, a reduction of phenytoin dose might be required. ${ }^{16}$

\section{Valproate and levetiracetam}

A concomitant administration of ESL with valproate or levetiracetam does not influence the exposure to eslicarbazepine. ${ }^{12-14}$

\section{Interaction with other non-AEDs \\ Oral contraceptives}

Administration of ESL 1,200 mg once daily in female patients who are taking oral contraceptives led to an average decrease of $37 \%$ and $42 \%$ in systemic exposure to levonorgestrel and ethinyl estradiol, respectively, most likely caused by an induction of CYP3A4. Consequently, women of childbearing age must use adequate contraception during treatment with ESL. ${ }^{29}$

\section{Simvastatin}

A study in healthy subjects showed an average decrease of $50 \%$ in systemic exposure to simvastatin, if coadministered with ESL $800 \mathrm{mg}$ once daily, most likely caused by an induction of CYP3A4. ${ }^{16}$ It may be necessary to increase the dose of simvastatin, when administered. ${ }^{12-14}$

\section{Rosuvastatin}

When coadministered with ESL 1,200 mg once daily, there is a mean reduction in systemic exposure to $36 \%-39 \%$. Since the correlation between exposure and pharmacological activity is poorly understood, it is advisable to monitor response to therapy (eg, cholesterol levels). ${ }^{12-14}$

\section{Warfarin}

Coadministration of 1,200 mg once daily ESL with warfarin led to a small (23\%) but statistically significant decrease in the S-warfarin. There was no effects on the R-warfarin 
pharmacokinetics or on coagulation. However, due to interindividual variability of the interaction, special attention on monitoring of international normalized ratio should be performed the first weeks after initiation or ending concomitant treatment of warfarin and ESL. ${ }^{12-14}$

\section{Digoxin}

No effect has been reported of ESL 1,200 mg once daily on digoxin pharmacokinetics. ${ }^{12-14}$

\section{Efficacy}

\section{ESL as adjunctive therapy}

\section{Phase II study}

Efficacy and safety of ESL as add-on treatment in adults with uncontrolled POS were first demonstrated in a Phase II trial. ${ }^{30}$ This study was conducted in refractory patients with $\geq 4$ POS per month in spite of treatment with one or two AEDs. Patients were randomly assigned to one of three groups: ESL once daily $(n=50)$, twice daily $(n=46)$, or placebo $(n=47)$. The daily dose was titrated from $400 \mathrm{mg}$ to $800 \mathrm{mg}$ and to 1,200 $\mathrm{mg}$ at 4-week intervals. The study design consisted of a 12 -week treatment period followed by a 1-week tapering off. The proportion of responders (patients with $\mathrm{a} \geq 50 \%$ seizure reduction) was compared between active treatment and placebo groups: a statistically significant difference was observed only between once daily and placebo groups ( $54 \%$ vs $28 \%$; $P=0.008$ ), while no significant difference was noted between the twice daily (41\%) and placebo groups $(P=0.12)$. Moreover, authors performed a secondary analysis according to the treatment periods of weeks $1-4$ (400 mg/day or placebo) and weeks 5-8 (800 mg/day or placebo). A significantly higher proportion of responders in weeks 5-8 was found in the once daily group than in both twice daily and placebo groups $(58 \%, 33 \%$, and $38 \%$ respectively, $P=0.022$ and $P=0.022$ ). At all 4 -week periods, the once daily group showed a significantly higher reduction in the seizure number than the placebo group $(P=0.037, P=0.018$, and $P=0.002$, respectively). During the treatment period, the number of seizure-free patients increased in both ESL treatment groups with a statistical significance reached during the last 4 weeks (1,200 mg/day) in both once daily and twice daily groups as compared to the placebo. ${ }^{30}$ Based on results coming from this clinical study, ESL once daily administration regimen has been used in all subsequent trials.

\section{Phase III studies}

To date, four Phase III studies have been performed in order to assess efficacy and safety of ESL as adjunctive therapy in patients with POS not well controlled by their current AEDs (2093-301, ${ }^{30} 2093-302,{ }^{31} 2093-303,{ }^{32}$ and 2093-304). ${ }^{33}$

Demographic characteristics and main results of the four Phase III double-blind, add-on studies are shown in Table 1. Patients had been diagnosed with simple or complex POS for $\geq 12$ months $^{31-33}$ or epilepsy for $\geq 12$ months with POS for $\geq 4$ months. ${ }^{34}$ All of them were experiencing POS despite being treated with a stable dose of one to two ${ }^{31,33,34}$ or one to three ${ }^{32}$ concomitant AEDs (except OXC). After an observational 8-week baseline period, in the double-bind phase patients were randomized to receive ESL $400 \mathrm{mg} /$ day, ${ }^{31,32}$ $800 \mathrm{mg} /$ day, $1,200 \mathrm{mg} /$ day or placebo. The study design

Table I Main results of the primary endpoints of the double-blind, add-on studies, case-control Phase III studies

\begin{tabular}{|c|c|c|c|c|}
\hline References & $\begin{array}{l}\text { Number of } \\
\text { concomitant AEDs }\end{array}$ & $\begin{array}{l}\text { Randomization groups } \\
\text { (number of patients) }\end{array}$ & $\begin{array}{l}\text { Responder rate } \\
\text { (\% of patients) }\end{array}$ & $\begin{array}{l}\text { Median relative reduction } \\
\text { in seizure frequency (\%) }\end{array}$ \\
\hline \multirow[t]{4}{*}{$\overline{\text { Elger et al }}{ }^{31}$} & 1 to 2 & ESL $400 \mathrm{mg}$ od (100 pts) & 23 & 26 \\
\hline & & ESL $800 \mathrm{mg}$ od (98 pts) & $34 *$ & $36 *$ \\
\hline & & ESL I,200 mg od (102 pts) & $43 * * *$ & $45 * *$ \\
\hline & & Placebo (I02 pts) & 20 & 16 \\
\hline \multirow[t]{4}{*}{ Ben-Menachem et $\mathrm{a}^{32}$} & I to 3 & ESL $400 \mathrm{mg}$ od (96 pts) & 17 & 18.7 \\
\hline & & ESL $800 \mathrm{mg}$ od (I0I pts) & $40 * * *$ & $32.6 * * *$ \\
\hline & & ESL I,200 mg od (98 pts) & $37 * * *$ & $32.8^{* * *}$ \\
\hline & & Placebo (100 pts) & 13 & 0.8 \\
\hline \multirow[t]{3}{*}{ Gil-Nagel et al ${ }^{33}$} & 1 to 2 & ESL $800 \mathrm{mg}$ od (85 pts) & 34.5 & $37.9 * * *$ \\
\hline & & ESL I,200 mg od (80 pts) & $37.7 *$ & $41.9 * * *$ \\
\hline & & Placebo (88 pts) & 22.6 & 17 \\
\hline \multirow[t]{3}{*}{ Sperling et $\mathrm{al}^{34}$} & I to 2 & ESL $800 \mathrm{mg}$ od (2I5 pts) & 30.5 & 29.7 \\
\hline & & ESL I,200 mg od (205 pts) & $42.6 * * *$ & $35.6^{*}$ \\
\hline & & Placebo (220 pts) & 23.1 & 21.8 \\
\hline
\end{tabular}

Notes: $* P<0.05 ; * * P<0.01 ; * * * P<0.001$ vs placebo

Abbreviations: ESL, eslicarbazepine acetate; AEDs, antiepileptic drugs; pts, patients; od, once daily. 
consisted of a 2 -week titration period followed by a 12-week maintenance period ${ }^{31,33,34}$ or 14-week duration trial in which all patients started at their full maintenance dose, except for those in the ESL $1,200 \mathrm{mg}$ /day group with dose titration during the first 2 weeks. ${ }^{32}$

The primary endpoint of all Phase III trials was decreased seizure frequency (standardized to a frequency per 4 weeks). Overall, in patients with POS, adjunctive therapy with ESL led to a significant decrease in standardized seizure frequency. According to randomized groups, in trials 2093-301, ${ }^{31} 2093-302,{ }^{32}$ and 2093-303, ${ }^{33}$ the standardized seizure frequency was significantly lower in both ESL 800 and $1,200 \mathrm{mg} /$ day groups than in the placebo group. In trial 2093-304, ${ }^{34}$ significance was reached only in the ESL 1,200 mg/day group; however, as noted by the authors, this could be explained by the relatively high responder rate in placebo treated patients. Responder rate (defined as a $\geq 50 \%$ reduction in standardized seizure frequency) was significantly higher in the ESL 1,200 $\mathrm{mg}$ /day group than in placebo recipients in all four studies, with a significance also observed in the ESL $800 \mathrm{mg}$ /day group compared to placebo in trials 2093-301 ${ }^{31}$ and 2093-302. ${ }^{32}$ Moreover, results of a further analysis of patients referred to the 2093-304 study $^{34}$ have been published. During the trial, the Seizure Severity Questionnaire, a tool developed in order to quantify the severity of seizures over time, was administered at baseline and post-ESL treatment. Authors concluded that ESL led to clinically meaningful, dose-dependent reduction in seizure severity, as measured by Seizure Severity Questionnaire scores. ${ }^{35}$

A Cochrane review including four trials $(1,146$ participants) ${ }^{30-33}$ showed that the overall relative risk with $95 \%$ confidence interval (CI) for a $50 \%$ or greater reduction in seizure frequency was 1.86 (95\% CI 1.46 to 2.36 ). Dose regression analysis showed that ESL reduced seizure frequency with an increase in efficacy with increasing doses of ESL. ESL was significantly associated with seizure freedom (relative risk 3.04, 95\% CI 1.44 to 6.42). ESL reduces seizure frequency as an add-on treatment in drug-resistant partial epilepsy. ${ }^{36}$

\section{Pooled analysis}

Data from the first three Phase III studies (1,049 patients) have been pooled and analyzed: ${ }^{37}$ seizure frequency was significantly reduced with ESL $800 \mathrm{mg} /$ day $(P<0.0001)$ and $1,200 \mathrm{mg} /$ day $(P<0.0001)$ groups compared to placebo (respectively, 35\%, 39\%, and 15\%). The responder rate was significantly higher in the ESL 800 (36\%) and 1,200 mg/day
(44\%) groups than in the placebo group (22\%). Moreover, ESL was more efficacious than placebo regardless of sex, geographic region, epilepsy duration, age at time of diagnosis, seizure type, and number and type of concomitant AEDs. ${ }^{37}$

\section{Long-term extension studies}

Subsequently, 1-year, open-label, extension studies ${ }^{38,39}$ have been performed from the Phase III trials 2093-301 ${ }^{33}$ and 2093-302. ${ }^{32}$ All patients received an ESL starting dose of $800 \mathrm{mg}$ once daily, for 4 weeks followed by an individualized titration to between $400 \mathrm{mg} /$ day and 1,200 mg/day. The percentage of patients completing 1 year of treatment was $76.6 \%$ and $68.6 \%$. In both studies, ESL showed sustained efficacy as add-on treatment in adults with refractory POS. Compared to baseline, median seizure frequency was $32 \%-39 \% 38,39$ during the first 4 weeks and between $37 \%-48 \%{ }^{38,39}$ and $39 \%-56 \%{ }^{38,39}$ thereafter. The responder rate was $37 \%-41 \%{ }^{39}$ during weeks $1-4$ and thereafter ranged between $38 \%-48 \%{ }^{38,39}$ and $42 \%-53 \%{ }^{38}$ per 12 -week interval. The proportion of seizure-free patients per 12-week interval ranged between $5 \%-8.7 \% 0^{38,39}$ and $11 \%-12.5 \% .^{38,39}$ In addition, in both studies significant improvements in quality of life and depressive symptoms have been observed in the long run.

\section{Clinical practice studies}

Post-authorization studies aimed at retrospectively evaluating, in a clinical practice setting, the long-term efficacy of ESL as add-on therapy, have confirmed the encouraging results of previous studies. ${ }^{40,41}$

Efficacy and safety were retrospectively evaluated over $1-2^{40,41}$ years in patients with a diagnosis of epilepsy and focal seizures who were treated according to clinical practice with ESL. At the end of the 1-year study 237 patients (72.5\%) were still on ESL, ${ }^{40}$ while in the 2-year study 56 patients $(36.8 \%)$ dropped out of treatment; and retention rates as estimated by Kaplan-Meyer curves were $82.9 \%, 71.3 \%, 65.1 \%$, and $62.8 \%$, respectively, at $6,12,18$, and 24 months. ${ }^{41}$

Results of the 1-year retrospective study showed that at 3,6 , and 12 months, the responder rates were $46.3 \%, 57.9 \%$, and $52.5 \%$; and $21.0 \%, 28.0 \%$, and $25.3 \%$ of patients were seizure-free. ${ }^{40}$

In the 2-year retrospective, observational study after 6 , 12,18 , and 24 months' follow-up the responder rates were $25.7 \%, 25.7 \%, 19.0 \%$, and $17.1 \%$, respectively and favorable global clinical impression (based on the mean seizure frequency of the previous 3 months) rates were $27.7 \%$, $19.7 \%, 17.8 \%$, and $16.5 \% .^{41}$ 
Despite the fact that in the latter study the responder rates were lower than those of previous studies, authors concluded that ESL appears to be a clinically useful add-on AED even in a very refractory group of patients. ${ }^{41}$

\section{Pediatric study}

ESL is currently not recommended in children. To date there has been one open-label, clinical study performed in pediatric patients highly refractory to treatment distributed into three age groups: 2-6years (group 1, eleven patients), 7-11 years (group 2, eight patients), and 12-17 years (group 3, ten patients). In each age group, three different dosage regimens were studied: $5 \mathrm{mg} / \mathrm{kg} /$ day (weeks $1-4$ ), $15 \mathrm{mg} / \mathrm{kg} /$ day (weeks $5-8$ ), and $30 \mathrm{mg} / \mathrm{kg} /$ day or $1,800 \mathrm{mg} /$ day, whichever was less (weeks 9-12). Patients were treated with a stable dose of one to three concomitant AEDs. The study showed a clear dosedependent decrease in seizure frequency in group 1 (28.2\%, $24.8 \%$, and $40.6 \%$ with 5,15 , and $30 \mathrm{mg} / \mathrm{kg} /$ day, respectively) and group $3(17.1 \%, 31.7 \%$ and $43.1 \%, 11.7 \%, 5 \%$ and $12.2 \%)$, while, in group 2 , no significant changes were observed $(11.7 \%$, $5 \%$ and $12.2 \%$ with 5,15 , and $30 \mathrm{mg} / \mathrm{kg} /$ day, respectively). Despite the limited number of patients, authors concluded that ESL might have a promising role also in pediatric patients. ${ }^{42}$

\section{ESL as monotherapy}

\section{Phase III studies}

Based on these encouraging data on the efficacy of ESL as adjunctive therapy, two identically designed randomized, double-blind, "withdrawal to monotherapy" Phase III trials (studies $093-045^{43}$ and 093-046) ${ }^{44}$ have been performed in order to assess the efficacy and safety of ESL as monotherapy. Patients 16 years of age or older with POS medically uncontrolled by one to two AEDs were gradually converted to ESL monotherapy. After an 8-week baseline period for assessment of seizure frequency, patients were randomized 2:1 to receive ESL 1,600 mg or 1,200 mg once daily, historical data were used as control. ESL was titrated to the target dose during the first 2 weeks of double-blind treatment, after which, doses of baseline AEDs were reduced (by 50\% in the first 3 weeks, and to zero during the next 3 weeks). At the end of this conversion period, patients continued to receive their allocated ESL dose $(1,200$ or $1,600 \mathrm{mg})$ as monotherapy for 10 weeks. Primary efficacy endpoint was the exit rate for patients meeting at least one of five of the following prospective exit criteria (signifying worsening seizure control): 1) one episode of status epilepticus; 2 ) one secondary generalized partial seizure (for patients without generalized seizures during 6 months prior to screening); 3) two-fold increase from baseline in consecutive 28-day seizure rate; 4) two-fold increase from baseline in consecutive 2-day seizure rate; 5) worsening of seizures or increase in seizure frequency. Treatment was considered effective if the $95 \%$ upper confidence limit for the Kaplan-Meier estimated exit rate was below the lower limit of the pre-specified prediction interval (65.3\%) calculated from historical controls.

In both studies, results of the primary efficacy endpoint analysis demonstrated that the exit rates for patients who converted to ESL monotherapy were statistically lower than the exit rates observed in the placebo arms of the historical controls trials. The Kaplan-Meier estimated exit rate was 12.8\% $(95 \% \text { CI } 7.5 \%-21.5 \%)^{44}$ and $28.7 \%(95 \% \text { CI } 21.2 \%-38.1 \%)^{43}$ for ESL $1,600 \mathrm{mg}$ and $15.6 \%(8.1 \%-28.7 \%)^{44}$ and $44.4 \%$ $(32.5 \%-58.3 \%)^{43}$ for ESL $1,200 \mathrm{mg}$. Thus, the upper confidence limit for the Kaplan-Meier exit rates were below the $65.3 \%$ threshold calculated from the historical controls. Main results of the secondary endpoints are summarized in Table 2. Overall, there was a reduction in standardized seizure frequency from baseline across different seizure types and the improvements observed with ESL in several of the secondary

Table 2 Main results of the secondary endpoints of the double-blind, "withdrawal to monotherapy", historical control Phase III studies

\begin{tabular}{|c|c|c|c|c|c|}
\hline References & $\begin{array}{l}\text { Number of AEDs at } \\
\text { baseline (number of } \\
\text { patients, } \% \text { ) }\end{array}$ & $\begin{array}{l}\text { Randomization } \\
\text { groups (number of } \\
\text { patients) }\end{array}$ & $\begin{array}{l}\text { Pts meeting } \\
\text { seizure- related } \\
\text { exit criteria }{ }^{a} \text {, number } \\
\text { of patients }(\%)\end{array}$ & $\begin{array}{l}\text { Changes in SSF } \\
\text { from baseline, } \\
\text { median } \%\end{array}$ & $\begin{array}{l}\text { Responder rate } \\
\text { dose, \% (number } \\
\text { of patients) }\end{array}$ \\
\hline Jacobson et al, ${ }^{44} 2015$ & I AED (I I I pts, 64.5\%) & ESL I,200 mg (58 pts) & 7 pts (I2\%) & $-36.1 \%$ & $35.2 \%(19 \mathrm{pts})$ \\
\hline$(093-046)$ & 2 AEDs (6I pts, 35.5\%) & ESL I,600 mg (I l4 pts) & $\begin{array}{l}12 \text { pts }(10.5 \%) \\
\text { Total: } 19 \text { pts (13.3\%) }\end{array}$ & $-47.5 \%$ & $46.0 \%$ (46 pts) \\
\hline Sperling et al, ${ }^{43} 2015$ & I AED (I42 pts, 73.6\%) & ESL I,200 mg (65 pts) & 23 pts $(35 \%)$ & $-30.9 \%$ & $36.7 \%(22 \mathrm{pts})$ \\
\hline$(093-045)$ & 2 AEDs (5I pts, 26.4\%) & ESL I,600 mg (I 28 pts) & $\begin{array}{l}\text { I } 7 \text { pts }(13.2 \%) \\
\text { Total: } 40 \text { pts }(22.5 \%)\end{array}$ & $-41.5 \%$ & $39.8 \%$ (47 pts) \\
\hline
\end{tabular}

Notes: ${ }^{2}$ Exit criteria: I) one episode of status epilepticus; 2) one secondary generalized partial seizure (for patients without generalized seizures during 6 months prior to screening); 3) two-fold increase from baseline in consecutive 28-day seizure rate; 4) two-fold increase from baseline in consecutive 2-day seizure rate; 5) worsening of seizures or increase in seizure frequency (as judged by investigator).

Abbreviations: ESL, eslicarbazepine acetate; SSF, standardized seizure frequency (seizure frequency is standardized to a 28-day frequency); AEDs, antiepileptic drugs; pts, patients. 
efficacy endpoints (proportion of seizure-free patients during monotherapy, reduction in standardized seizure frequency, and responder rate) are consistent with the suggestion that ESL monotherapy is a potential treatment option for patients with POS. Supported by data from these two pivotal Phase III clinical trials, ESL achieved FDA approval as a monotherapy indication for patients with POS. ${ }^{14}$

\section{Tolerability and safety ESL as adjunctive therapy}

\section{Phase III studies}

According to the four Phase III studies analyzed, ESL is considered safe and well tolerated when used at the dosage of 800 and 1,200 $\mathrm{mg}$ once daily as add-on treatment in adults with $\geq 4$ POS. ${ }^{31-34}$ The most frequent adverse events (AEs) involved more than $10 \%$ of patients ${ }^{31-33}$ and were: dizziness, headache, diplopia, ${ }^{31-34}$ somnolence, nausea, abnormal coordination, vomiting, blurred vision, and fatigue. ${ }^{32,33}$ A Cochrane review including four trials ${ }^{30-33}$ came to similar conclusions. ${ }^{36}$ Other AEs found in the four trials analyzed were ataxia, fatigue, somnolence, rash, headache, and vomiting. ${ }^{36}$

Psychiatric disorders were found in very few cases in all the trials, and consisted of depression, ${ }^{31,32}$ insomnia, irritability, ${ }^{31}$ agitation, and apathy. ${ }^{32}$

The incidence of rash, which is the most common idiosyncratic reaction with all AEDs, seems to be low in patients treated with ESL. This adverse effect occurred in approximately $1 \%-2.4 \%$ of patients in all Phase III ESL studies analyzed, and it was from mild to moderate intensity. ${ }^{31-34}$ Hyponatremia is another frequent AE with the use of dibenzazepines. Hyponatremia occurred in $0.6 \%$ to $1.5 \%$ of ESL treated patients, ${ }^{33,34}$ and $5.1 \%$ had a reduction in serum sodium values $>10 \mathrm{mEq} / \mathrm{L}$ during treatment, ${ }^{34}$ moreover there was a greater reduction in mean sodium values in the first 8 weeks of treatment and these values tended to stabilize after approximately 2 months of treatment. ${ }^{34}$

One case of severe hyponatremia (sodium level $<125 \mathrm{mmol} / \mathrm{L}$ ) was reported at the end of the maintenance period in a 31-year-old patient, who was in the ESL $800 \mathrm{mg}$ group and was treated with CBZ 1,000 mg/day concomitantly, who presented a value of $123 \mathrm{mmol} / \mathrm{L}$ at the end of the maintenance period; levels returned to normal values after the tapering-off period. ${ }^{31}$ Hyponatremia was reported as an AE leading to discontinuation in $1.4 \%$ of patients taking ESL $1,200 \mathrm{mg}$, but in no one taking placebo or ESL $800 \mathrm{mg} .{ }^{34}$

Regarding other clinical laboratory parameters like glucose and lipid plasma values, liver functionality and other parameters like vital signs, body weight, or electrocardiogram (ECG): no clinically relevant findings were found. ${ }^{31-34}$
Treatment emergent adverse events (TEAEs) involved more than $10 \%$ of patients in all studies and were usually from mild to moderate intensity. ${ }^{31-34}$ The most common TEAEs reported were: dizziness, headache, somnolence, ${ }^{31-34}$ nausea, ${ }^{32-34}$ diplopia, and abnormal coordination, vomiting, blurred vision, and fatigue that occurred in $5 \%$ of patients. ${ }^{32}$ TEAEs increased with increasing ESL dose, ${ }^{31-34}$ and mostly occurred during the first 41 days of treatment. ${ }^{32,33}$ TEAEs leading to discontinuation in more than $2 \%^{34}$ or $3 \%{ }^{33}$ of patients taking ESL were: dizziness, ${ }^{31,33,34}$ nausea, ${ }^{31,33,34}$ somnolence, ${ }^{31,34}$ abnormal coordination, ${ }^{33}$ diplopia, ${ }^{31}$ vomiting, ataxia, and dysarthria. ${ }^{34}$ One study reported hyponatremia as a TEAE in four ESL patients of 503 patients enrolled at baseline (ESL 1,200 mg: $\mathrm{n}=2$; ESL $800 \mathrm{mg}$ : $\mathrm{n}=1$; ESL $400 \mathrm{mg}$ : $\mathrm{n}=1$ ), but for no placebo patients. ${ }^{32}$ Three patients were also taking $\mathrm{CBZ}$ as concomitant AED. Severe TEAEs occurred with a minimal incidence in patients during the treatment period ${ }^{31-34}$ and did not increase with increasing ESL dose. ${ }^{31}$ Most common severe TEAEs were: dizziness and vertigo that occurred in more than $2 \%$ of all the severe TEAEs. ${ }^{34}$ It has been reported that a case of cerebellar syndrome (in the ESL 1,200 mg group) led to study discontinuation. ${ }^{33}$ In one study, two deaths occurred during the double-blind period: a 29-year-old Caucasian woman randomized to placebo died from acute respiratory failure with pneumonia; and a 27-year-old Caucasian man randomized to ESL $800 \mathrm{mg}$ was found dead during the titration phase, because of status epilepticus. ${ }^{34}$

Meta-analysis from clinical, double-blind, placebocontrolled trials aimed at evaluating the overall tolerability of ESL, OXC, and LCM, showed that the AEs were clearly dose-dependent. Comparison data between the AEDs revealed that ESL and LCM were safer and less associated with neurological AEs than OXC. ${ }^{45}$

\section{Pooled analysis}

Data from the first three Phase III studies have been pooled and analyzed, and confirmed that the majority of TEAEs were of mild to moderate intensity, and incidence of AEs and AEs leading to discontinuation were dose-dependent. ${ }^{37}$ AEs occurred mainly during the first weeks of treatment, with no difference between groups after 6 weeks. There were severe TEAEs, but no dose-dependent trend was observed. Starting dose was important because patients who started at ESL $400 \mathrm{mg}$ reported $35 \%-45 \%$ of TEAEs; patients who began treatment at ESL $600 \mathrm{mg}$ had an incidence of $51 \%$ of TEAEs, and patients who started with ESL $800 \mathrm{mg}$ presented incidence of $73 \%-78 \%$. TEAEs were higher in women $(68.3 \%)$ treated with ESL than in men $(59.2 \%)$, and 
with respect to placebo (women $47.9 \%$; men $44.8 \%$ ). The number of AEDs (one or two) did not have an effect on the incidence of TEAEs, however, concomitant treatment with CBZ showed an increase in some TEAEs such as diplopia ( $11.4 \%$ vs $2.4 \%$ of patients not treated with CBZ), abnormal coordination (6.7\% vs $2.7 \%)$, and dizziness $(30.0 \%$ vs $11.5 \%$ ). Hyponatremia occurred in $0.5 \%$ of ESL $400 \mathrm{mg}$ group; $0.7 \%$ of ESL $800 \mathrm{mg}$ group; and $0.4 \%$ of ESL 1,200 $\mathrm{mg}$ group. No clinically relevant changes in body weight, vital signs, or ECG were observed. ${ }^{37}$

\section{Long-term extension studies}

One-year, open-label, extension studies ${ }^{38,39}$ have been performed from the Phase III trials ${ }^{31,32}$ and they found that from 51\% to $59.7 \% 0^{38,39}$ of patients reported at least one TEAE. The majority of TEAEs were mild or moderate, and the most frequent (occurring in $\geq 10 \%$ of patients) were headache $\left(10.2 \%{ }^{38}\right.$ and $15.7 \%),{ }^{39}$ dizziness $\left(10.2 \%{ }^{38}\right.$ and $\left.26.5 \%\right),{ }^{39}$ diplopia $(5.4 \%),{ }^{38}$ somnolence $(12 \%),{ }^{39}$ and nasopharyngitis $(5.1 \%) .{ }^{38}$ In the first 4 weeks of therapy, the majority of AEs occurred, and from $3.5 \%{ }^{38}$ to $11.4 \%{ }^{39}$ of patients discontinued for this reason. Incidence of TEAEs decreased over 1 year of therapy. ${ }^{38}$ Sodium plasma concentrations were usually within the therapeutic range. No relevant laboratory parameters were altered: blood pressure, heart rate, and laboratory parameters were normal. ${ }^{39}$

\section{Clinical practice studies}

Post-authorization studies aimed to evaluate retrospectively, the long-term tolerability of ESL as add-on therapy, confirming that ESL was well tolerated in a real-world setting. ${ }^{40,41}$ The most frequent AEs were dizziness, nausea, and somnolence and they occurred in $40.7 \%{ }^{40}$ to $42.1 \%{ }^{41}$ of patients at $12^{40}$ and $24^{41}$ months of treatment. From $16.2 \%{ }^{40}$ to $50.0 \%{ }^{41}$ of patients discontinued at $12^{40}$ and $24^{41}$ months of therapy due to AEs. All the AEs were mild to moderate. Hyponatremia was reported in $2.7 \%$ of patients and was symptomatic in one patient who presented with transient confusion that was resolved after ESL discontinuation. ${ }^{40}$ In one case there was a mild and transient elevation in AST/ALT levels but this did not lead to discontinuation. No other laboratory abnormalities were reported. ${ }^{40}$ Severe AEs were: three cases of rash and another of bicytopenia (lympho-cytopenia and thrombocytopenia). ${ }^{41}$ Approximately $56.0 \%$ of AEs appeared in the first 6 months of ESL treatment. ${ }^{41}$

\section{Pediatric study}

The pediatric open-label, clinical study performed in patients highly refractory to treatment showed that 54 AEs (drug related or not) were reported in 21 patients, and the frequency was dose-dependent. All the AEs were mild or moderate and were: somnolence (three patients), vomiting (two patients), diplopia (two patients), dizziness (one patient), and one case of equilibrium trouble. All these AEs resolved without therapy discontinuation. There were two cases of severe intensity AEs (seizure worsening leading to patient withdrawal by the study). There were no deaths and no clinically significant changes in laboratory parameters (vital signs, physical examination, and ECG parameters). ${ }^{42}$

\section{ESL as monotherapy}

\section{Phase III studies}

Two randomized, double-blind, "withdrawal to monotherapy" Phase III trials ${ }^{43,44}$ have been performed in order to assess the efficacy and safety of ESL as monotherapy. In these studies, $89 \%{ }^{43}$ and $67 \%{ }^{44}$ of the treated patients experienced more than one TEAE (ESL 1,600 mg: $91 \%{ }^{43}$ and 60\%, ${ }^{44}$ ESL 1,200 mg: $85 \%{ }^{43}$ and $\left.71 \%\right) .{ }^{44}$ The most commonly reported TEAEs were dizziness, headache, fatigue, somnolence, nausea, nasopharyngitis, vomiting, and blurred vision. TEAEs were reported more often during the titration period $\left(72 \%{ }^{43}\right.$ and $42 \%)^{44}$ than during the AED conversion $\left(61 \%{ }^{43} \text { and } 37 \%\right)^{44}$ and monotherapy periods $\left(49 \%{ }^{43}\right.$ and $\left.38 \%\right) .{ }^{44}$ Most of TEAEs were mild or moderate, but $8.8 \%{ }^{43}$ had $\geq 1$ severe TEAEs. Treatment emergent serious AEs (SAEs) occurred in $6.2 \%{ }^{44}$ of patients. In one study, ${ }^{43}$ the only SAE reported for ESL $1,200 \mathrm{mg}$ was atrial flutter (which occurred prior to dosing with ESL), while SAEs were reported by $7 \%$ for ESL 1,600 mg group, and 1.7\% for ESL 1,200 mg group and were: ankle fracture, post-concussion syndrome, tibia fracture, hyponatremia, complex partial seizures, partial seizures with secondary generalization, syncope, spontaneous abortion, drug rash with eosinophilia, and systemic symptoms. Most SAEs occurred during the AED conversion period. ${ }^{44}$ However, these TEAEs and SAEs were not frequent during the trial. Nine percent ${ }^{44}$ to $16 \%{ }^{43}$ of patients discontinued the trial due to a TEAE (ESL 1,600 mg: $12 \%{ }^{44}$ to $18 \%$; ${ }^{43}$ ESL 1,200 mg: $3 \%{ }^{44}$ to $12 \%$. ${ }^{43}$ Hyponatremia, complex partial seizures, and pruritic rash were the TEAEs that most frequently led to discontinuation. ${ }^{43}$

\section{Dosage and administration}

ESL is indicated in the US (Aptiom ${ }^{\mathrm{TM}}$ ) for both monotherapy and adjunctive treatment of adults aged $\geq 18$ years with POS $^{13,14}$ and in the EU (Zebinix ${ }^{\mathrm{TM}}$ ) as adjunctive therapy in adults aged $\geq 18$ years with POS with or without secondary generalization. ${ }^{12}$ 
ESL should be started at a dosage of $400 \mathrm{mg}$ once daily. After $1^{12,46}$ or 2 weeks, ${ }^{11}$ the dosage should be increased to $800 \mathrm{mg}$ once daily (recommended maintenance dosage). Maximum recommended maintenance dosage is $1,200 \mathrm{mg}$ once daily (after a minimum of 1 week at $800 \mathrm{mg}$ once daily). Patients with moderate to severe renal impairment should start the treatment at $200 \mathrm{mg}$ once daily, after 2 weeks, the dosage may be increased to $400 \mathrm{mg}$ once daily. Maximum recommended maintenance dosage is $600 \mathrm{mg}$ once daily. ${ }^{12,46}$ ESL is available in four tablet strengths $(200 \mathrm{mg}, 400 \mathrm{mg}$, $600 \mathrm{mg}$, and $800 \mathrm{mg}$ ), which can be taken whole or crushed, with or without food.

\section{Conclusion}

Over the last years, a new sodium-channel blocker, ESL has been licensed as adjunctive therapy in adults with refractory POS, with or without secondary generalization, ${ }^{12,13}$ and as monotherapy in people who initiate treatment for the first time or convert from other AEDs. ${ }^{14}$ Despite the fact that the exact mechanism of anticonvulsant action is still unclear, ESL can be considered as an advancement in terms of efficacy and tolerability. ${ }^{47,48}$ Moreover, its pharmacokinetic profile showed that ESL is associated with few clinically relevant drug interactions ${ }^{12-14}$ and has a long elimination half-life, ${ }^{25}$ permitting a convenient once daily administration regimen, as definitively demonstrated by clinical studies. ${ }^{30}$ This once daily drug regimen might lead to increased adherence, resulting in an improvement in patient outcomes. ${ }^{9}$ Adjunctive therapy with ESL 800 or 1,200 mg/day led to a significant decrease in the standardized seizure frequency in adults with refractory POS, according to the results of Phase III trials. ${ }^{31-34}$ According to the results of 1-year extension studies, ESL's efficacy was maintained over time, giving long-term improvements in quality of life. ${ }^{36,37} \mathrm{ESL}$ 's efficacy has been proven also as a monotherapy by randomized, double-blind, historical control Phase III studies, suggesting that ESL monotherapy is a potential treatment option for patients with POS. ${ }^{43,44}$

Overall, ESL was generally well tolerated in patients with POS. The most commonly reported AEs include dizziness, somnolence, headache, nausea, diplopia, and vomiting. AEs can be minimized by appropriate titration of the ESL dosage, as suggested by the FDA and EMA. ${ }^{12,46}$ Despite the overall paucity of data, also in pediatric patients, ESL showed encouraging results in terms of efficacy and tolerability, also in children. ${ }^{42}$

In conclusion, ESL seems to have overcome some drawbacks of the previous AEDs, suggesting a major role of ESL in the management of POS for both new onset and refractory cases, either as monotherapy or as adjunctive treatment.

\section{Disclosure}

The authors report no conflicts of interest in this work. No funding has been received for this work.

\section{References}

1. who.int [homepage on the Internet]. Epilepsy. Fact sheet $\mathrm{N}^{\circ} 999$ World Health Organization; 2016 [updated February 2016]. Available from: http://www.who.int/mediacentre/factsheets/fs999/en/. Accessed April 9, 2016.

2. Kwan P, Schachter SC, Brodie MJ. Drug-resistant epilepsy. N Engl J Med. 2011;365(10):919-926.

3. Semah F, Picot MC, Adam C, et al. Is the underlying cause of epilepsy a major prognostic factor for recurrence? Neurology. 1998;51(5): $1256-1262$.

4. Franco V, French JA, Perucca E. Challenges in the clinical development of new antiepileptic drugs. Pharmacol Res. 2016;103:95-104.

5. Perucca E, Tomson T. The pharmacological treatment of epilepsy in adults. Lancet Neurol. 2011;10(5):446-456.

6. Perucca P, Gilliam FG. Adverse effects of antiepileptic drugs. Lancet Neurol. 2012;11(9):792-802.

7. Beghi E, Messina P, Pupillo E, et al. Satisfaction with antiepileptic drugs in children and adolescents with newly diagnosed and chronic epilepsy. Epilepsy Res. 2012;100(1-2):142-151.

8. Witt JA, Elger CE, Helmstaedter C. Which drug-induced side effects would be tolerated in the prospect of seizure control? Epilepsy Behav. 2013;29(1):141-143.

9. Faught E. Adherence to antiepilepsy drug therapy. Epilepsy Behav. 2012;25(3):297-302.

10. Benes J, Parada A, Figueiredo AA, et al. Anticonvulsant and sodium channel-blocking properties of novel 10,11-dihydro-5H-dibenz[b,f] azepine-5-carboxamide derivatives. J Med Chem. 1999;42(14): 2582-2587.

11. Almeida L, Potgieter JH, Maia J, Potgieter MA, Mota F, Soares-da-Silva P. Pharmacokinetics of eslicarbazepine acetate in patients with moderate hepatic impairment. Eur J Clin Pharmacol. 2008;64(3):267-273.

12. European Medicines Agency [homepage on the Internet]. Zebinix (eslicarbazepine acetate) European public assessment reports. EMA; 2009. Available from: http://www.ema.europa.eu/ema/index.jsp?curl=pages/ medicines/human/medicines/000988/human_med_001172. jsp\&mid=WC0b01ac058001d124. Accessed April 10, 2016.

13. Sunovion Pharmaceuticals Inc. Sunovion Pharmaceuticals Inc Announces FDA Approval of Aptiom ${ }^{\circledR}$ (eslicarbazepine acetate) as OnceDaily Adjunctive Treatment of Partial-Onset Seizures [press release]. Marlborough, MA: Sunovion Pharmaceuticals Inc.; 2013. Available from: http://www.sunovion.com/news/pressReleases/20131108.pdf. Accessed April 10, 2016.

14. Sunovion Pharmaceuticals Inc. Sunovion Pharmaceuticals Inc Announces FDA Approval of a New Indication for Aptiom ${ }^{\circledR}$ (eslicarbazepine acetate) as Monotherapy for Partial Onset Seizures [press release]. Marlborough, MA: Sunovion Pharmaceuticals Inc.; 2015. Available from: http://www.sunovion.com/news/pressReleases/20150828.pdf. Accessed April 10, 2016.

15. Almeida L, Soares-de Silva P. Eslicarbazepine acetate (BIA 2-093). Neurotherapeutics. 2007;4(1):88-96.

16. Bialer M, Soares-da-Silva P. Pharmacokinetics and drug interactions of eslicarbazepine acetate. Epilepsia. 2012;53(6):935-946.

17. Bonifácio MJ, Sheridan RD, Parada A, Cunha RA, Patmore L, Soares-da-Silva P. Interaction of the novel anticonvulsant, BIA 2-093, with voltage-gated sodium channels: comparison with carbamazepine. Epilepsia. 2001;42(5):600-608. 
18. Soares-da-Silva P, Pires N, Bonifácio MJ, et al. Eslicarbazepine acetate for the treatment of focal epilepsy: an update on its proposed mechanisms of action. Pharmacol Res Perspect. 2015;3(2):e00124.

19. Araújo IM, Ambrósio AF, Leal EC, et al. Neurotoxicity induced by antiepileptic drugs in cultured hippocampal neurons: a comparative study between carbamazepine, oxcarbazepine, and two new putative antiepileptic drugs, BIA 2-024 and BIA 2-093. Epilepsia. 2004;45(12): 1498-1505.

20. Doeser A, Dickhof G, Reitze M, et al. Targeting pharmacoresistant epilepsy and epileptogenesis with a dual-purpose antiepileptic drug. Brain. 2015;138(Pt 2):371-387.

21. Brown DA, Passmore GM. Neural KCNQ (Kv7) channels. $\mathrm{Br} \mathrm{J}$ Pharmacol. 2009;156(8):1185-1195.

22. Maia J, Vaz-da-Silva M, Almeida L, et al. Effect of food on the pharmacokinetic profile of eslicarbazepine acetate (BIA 2-093). Drugs $R D$. 2005;6(4):201-206.

23. Almeida L, Falcão A, Maia J, Mazur D, Gellert M, Soares-da-Silva P. Single-dose and steady-state pharmacokinetics of eslicarbazepine acetate (BIA 2-093) in healthy elderly and young subjects. $J$ Clin Pharmacol. 2005;45(9):1062-1066.

24. Falcao A, Fuseau E, Nunes T, Almeida L, Soares-da-Silva P. Pharmacokinetics, drug interactions and exposure-response relationship of eslicarbazepine acetate in adult patients with partial-onset seizures: population pharmacokinetic and pharmacokinetic/pharmacodynamics analyses. CNS Drugs. 2012;26(1):79-91.

25. Perucca E, Elger C, Halász P, Falcão A, Almeida L, Soares-da-Silva P. Pharmacokinetics of eslicarbazepine acetate at steady-state in adults with partial-onset seizures. Epilepsy Res. 2011;96(1-2):132-139.

26. Falcão A, Maia J, Almeida L, et al. Effect of gender on the pharmacokinetics of eslicarbazepine acetate (BIA 2-093), a new voltagegated sodium channel blocker. Biopharm Drug Dispos. 2007;28(5): 249-256.

27. Almeida L, Nunes T, Sicard E, Rocha JF, et al. Pharmacokinetic interaction study between eslicarbazepine acetate and lamotrigine in healthy subjects. Acta Neurol Scand. 2010;121(4):257-264.

28. Nunes T, Sicard E, Almeida L, et al. Pharmacokinetic interaction study between eslicarbazepine acetate and topiramate in healthy subjects. Curr Med Res Opin. 2010;26(6):1355-1362.

29. Falcão A, Vaz-da-Silva M, Gama H, Nunes T, Almeida L, Soaresda-Silva P. Effect of eslicarbazepine acetate on the pharmacokinetics of a combined ethinylestradiol/levonorgestrel oral contraceptive in healthy women. Epilepsy Res. 2013;105(3):368-376.

30. Elger C, Bialer M, Cramer JA, Maia J, Almeida L, Soares-da-Silva P. Eslicarbazepine acetate: a double-blind, add-on, placebo-controlled exploratory trial in adult patients with partial-onset seizures. Epilepsia. 2007;48(3):497-504.

31. Elger C, Halász P, Maia J, et al. Efficacy and safety of eslicarbazepine acetate as adjunctive treatment in adults with refractory partial-onset seizures: a randomized, double-blind, placebo-controlled, parallel-group phase III study. Epilepsia. 2009;50(3):454-463.

32. Ben-Menachem E, Gabbai AA, Hufnagel A, Maia J, Almeida L, Soares-da-Silva P. Eslicarbazepine acetate as adjunctive therapy in adult patients with partial epilepsy. Epilepsy Res. 2010;89(2-3):278-285.

33. Gil-Nagel A, Lopes-Lima J, Almeida L, et al. Efficacy and safety of 800 and $1200 \mathrm{mg}$ eslicarbazepine acetate as adjunctive treatment in adults with refractory partial-onset seizures. Acta Neurol Scand. 2009;120(5): 281-287.

Neuropsychiatric Disease and Treatment

\section{Publish your work in this journal}

Neuropsychiatric Disease and Treatment is an international, peerreviewed journal of clinical therapeutics and pharmacology focusing on concise rapid reporting of clinical or pre-clinical studies on a range of neuropsychiatric and neurological disorders. This journal is indexed on PubMed Central, the 'PsycINFO' database and CAS,
34. Sperling MR, Abou-Khalil B, Harvey J, et al. Eslicarbazepine acetate as adjunctive therapy in patients with uncontrolled partial-onset seizures: Results of a phase III, double-blind, randomized, placebo-controlled trial. Epilepsia. 2015;56(2):244-253.

35. Gil-Nagel A, Elger C, Ben-Menachem E, et al. Efficacy and safety of eslicarbazepine acetate as add-on treatment in patients with focalonset seizures: integrated analysis of pooled data from double-blind phase III clinical studies. Epilepsia. 2013;54(1):98-107.

36. Halász P, Cramer JA, Hodoba D, et al. Long-term efficacy and safety of eslicarbazepine acetate: results of a 1-year open-label extension study in partial-onset seizures in adults with epilepsy. Epilepsia. 2010;51(10): 1963-1969.

37. Hufnagel A, Ben-Menachem E, Gabbai AA, Falcão A, Almeida L, Soares-da-Silva P. Long-term safety and efficacy of eslicarbazepine acetate as adjunctive therapy in the treatment of partial-onset seizures in adults with epilepsy: results of a 1-year open-label extension study. Epilepsy Res. 2013;103(2-3):262-269.

38. Cramer JA, Velez FF, Anastassopoulos KP, et al. Severity and burden of partial-onset seizures in a phase III trial of eslicarbazepine acetate. Epilepsy Behav. 2015;53:149-153.

39. Chang XC, Yuan H, Wang Y, Xu HQ, Zheng RY. Eslicarbazepine acetate add-on for drug-resistant partial epilepsy. Cochrane Database Syst Rev. 2011;(12):CD008907.

40. Villanueva V, Serratosa JM, Guillamón E, et al. Long-term safety and efficacy of eslicarbazepine acetate in patients with focal seizures: results of the 1-year ESLIBASE retrospective study. Epilepsy Res. 2014; 108(7):1243-1252.

41. Correia FD, Freitas J, Magalhães R, et al. Two-year follow-up with eslicarbazepine acetate: a consecutive, retrospective, observational study. Epilepsy Res. 2014;108(8):1399-1405.

42. Almeida L, Minciu I, Nunes T, et al. Pharmacokinetics, efficacy, and tolerability of eslicarbazepine acetate in children and adolescents with epilepsy. J Clin Pharmacol. 2008;48(8):966-977.

43. Sperling MR, Harvey J, Grinnell T, et al. Efficacy and safety of conversion to monotherapy with eslicarbazepine acetate in adults with uncontrolled partial-onset seizures: a randomized historical-control phase III study based in North America. Epilepsia. 2015;56(4):546-555.

44. Jacobson MP, Pazdera L, Bhatia P, et al. Efficacy and safety of conversion to monotherapy with eslicarbazepine acetate in adults with uncontrolled partial-onset seizures: a historical-control phase III study. BMC Neurol. 2015;15:46.

45. Zaccara G, Giovannelli F, Maratea D, Fadda V, Verrotti A. Neurological adverse events of new generation sodium blocker antiepileptic drugs. Meta-analysis of randomized, double-blinded studies with eslicarbazepine acetate, lacosamide and oxcarbazepine. Seizure. 2013;22(7): 528-536.

46. US Food and Drug Administration. Highlights of prescribing information: APTIOM ${ }^{\circledR}$ (eslicarbazepine acetate). FDA; 2013. Available from: http://www.accessdata.fda.gov/drugsatfda_docs/ label/2013/022416s000lbl.pdf. Accessed April 10, 2016.

47. Zaccara G, Giovannelli F, Cincotta M, Carelli A, Verrotti A. Clinical utility of eslicarbazepine: current evidence. Drug Des Devel Ther. 2015; 9:781-789.

48. Keating GM. Eslicarbazepine acetate: a review of its use as adjunctive therapy in refractory partial-onset seizures. CNS Drugs. 2014;28(7): 583-600.

and is the official journal of The International Neuropsychiatric Association (INA). The manuscript management system is completely online and includes a very quick and fair peer-review system, which is all easy to use. Visit http://www.dovepress.com/testimonials.php to read real quotes from published authors. 